\title{
Effect of Nonlinearity on PMD Compensation in a Single-Channel 10-Gb/s NRZ System
}

\author{
John Cameron ${ }^{*}, 1,2$ Xiaoyi Bao ${ }^{1}$ and Liang Chen $^{1}$ \\ ${ }^{I}$ Physics Department, University of Ottawa, Canada \\ ${ }^{2}$ Present Address: BTI Systems, Ottawa, Canada
}

\begin{abstract}
We simulate a 2000-km 10-Gb/s single-channel NRZ system with different polarization mode dispersion compensation (PMDC) techniques and compare the effectiveness of compensation with linear and nonlinear signal propagation. Significant differences are found between the results of PMDC with and without nonlinearity for all the compensation techniques that are examined. An analysis of PMDC based on linear propagation would not properly characterize PMDC in the nonlinear case. A three-stage compensator controlled by optimizing the signal eye opening is found to give large performance improvement with nonlinear propagation compared to single-stage PMDC.
\end{abstract}

\section{INTRODUCTION}

Compensation of polarization mode dispersion (PMD) has been an important area of research in high-capacity optical communications for more than a decade $[1,2]$. While the vast majority of published work on PMD compensation (PMDC) has considered propagation in the optical fiber to be linear, it has been demonstrated that in some systems the impact of fiber nonlinearity must be taken into account [3-8]. Most of the work on PMDC in nonlinear systems has focused on the effects of cross-phase modulation (XPM) in wavelength-division-multiplexed systems. XPM can cause polarization fluctuations on the time scale of the bit period which can seriously degrade the performance of PMDC [47].

There have been few publications examining the impact of single-channel nonlinearity on PMDC. Alzetta and Matsumoto [8] simulated PMDC in a dispersion-managed soliton (DMS) system and concluded that the signal degree of polarization (DOP) was less effective as a feedback control signal in the DMS system than in a linear system. Significant improvement in the compensation was found by maximizing the eye opening rather than DOP. Ibragimov et al. [3] simulated a $578-\mathrm{km} 10-\mathrm{Gb} / \mathrm{s} \mathrm{NRZ} \mathrm{system} \mathrm{with} \mathrm{propagation} \mathrm{of} \mathrm{a}$ single channel and showed that the interaction of nonlinearinduced chirp and higher-order PMD could, with proper alignment of the input state of polarization (SOP), increase the eye opening relative to the case where PMD was absent. It was concluded, therefore, that the system performance could be improved by dynamically controlling the input SOP to maximize the eye opening.

In this letter we investigate the effect of nonlinearity (self-phase modulation) in a single-channel $10-\mathrm{Gb} / \mathrm{s} \mathrm{NRZ}$ system with PMDC implemented at the receiver. We simulate a $2000-\mathrm{km}$ system and compare the performance of

\footnotetext{
*Address correspondence to this author at the BTI Systems, Ottawa, Canada; Tel: 613-248-9154, Ext. 386; Fax: 613-248-9156;

E-mail: jcameron@btisystems.com
}

PMDC under linear and nonlinear propagation with different compensator structures and feedback control methods. Clear differences are found in the results of PMDC with and without nonlinearity for all of the PMDC techniques that are examined.

\section{DESCRIPTION OF THE SIMULATIONS}

The basic system that is simulated consists of twenty amplified spans, with each span made up of $100 \mathrm{~km}$ of transmission fiber followed by a $5-\mathrm{km}$ long dispersion compensating fiber (DCF). DCFs $2 \mathrm{~km}$ in length are also placed at the transmitter and receiver sites for pre- and postcompensation of dispersion. A diagram of the system is given in Fig. (1). The single-channel $10 \mathrm{~Gb} / \mathrm{s}$ signal has an NRZ waveform with $10-90 \%$ rise and fall times of 23.6 ps and the bit pattern is a 64-bit De Bruijn sequence.

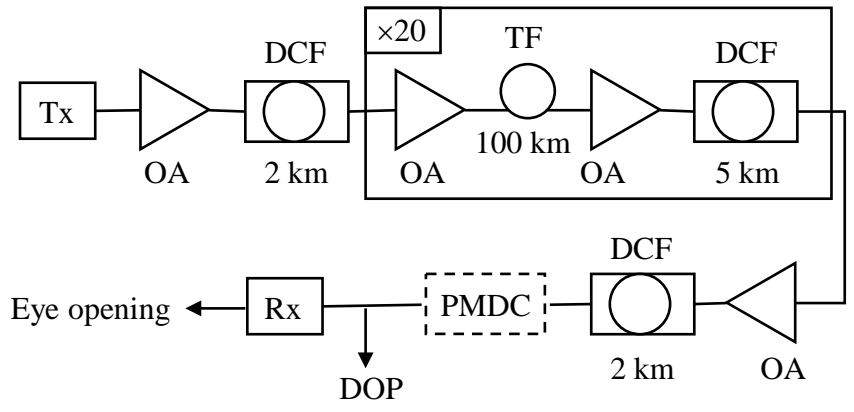

Fig. (1). System diagram. Tx: transmitter. OA: optical amplification. DCF: dispersion compensating fiber. TF: transmission fiber. $\mathrm{Rx}$ : receiver.

Two variants of the basic system are studied, which we refer to as systems A and B, and there are only two differences between them: 1) the dispersion map; and, 2) the mean value of the fiber differential group delay (DGD). The mean DGD values are 35 ps and 25 ps for systems $A$ and $B$, respectively. Details of the dispersion maps are summarized in Table 1. System A has $-200 \mathrm{ps} / \mathrm{nm}$ pre-compensation, 200 $\mathrm{ps} / \mathrm{nm}$ post compensation, and a residual dispersion in each span of $15 \mathrm{ps} / \mathrm{nm}$, giving a total net dispersion of $300 \mathrm{ps} / \mathrm{nm}$. 
System B has pre-compensation of $-100 \mathrm{ps} / \mathrm{nm}$, postcompensation of $100 \mathrm{ps} / \mathrm{nm}$, and full compensation in each span, giving zero total net dispersion.

Table 1. Summary of Fiber Dispersion Characteristics for System A and System B

\begin{tabular}{|c|c|c|c|c|}
\hline \multirow{2}{*}{ Fiber } & \multicolumn{2}{|c|}{ System A } & \multicolumn{2}{c|}{ System B } \\
\cline { 2 - 5 } & $\begin{array}{c}\mathbf{L} \\
(\mathbf{k m})\end{array}$ & $\begin{array}{c}\mathbf{D} \\
(\mathbf{p s} / \mathbf{n m}-\mathbf{k m})\end{array}$ & $\begin{array}{c}\mathbf{L} \\
(\mathbf{k m})\end{array}$ & $\begin{array}{c}\mathbf{D} \\
(\mathbf{p s} / \mathbf{n m}-\mathbf{k m})\end{array}$ \\
\hline \hline $\begin{array}{c}\text { Transmission } \\
\text { fiber spans }\end{array}$ & 100 & 6 & 100 & 6 \\
\hline DCF (inline) & 5 & -117 & 5 & -120 \\
\hline $\begin{array}{c}\text { DCF } \\
\text { (pre-compensation) }\end{array}$ & 2 & -100 & 2 & -50 \\
\hline $\begin{array}{c}\text { DCF } \\
\text { (post-compensation) }\end{array}$ & 2 & 100 & 2 & 50 \\
\hline
\end{tabular}

Propagation in the fiber is simulated with the coarse-step method [9]. The fiber is divided into $1-\mathrm{km}$ segments, with the birefringence constant over the length of a given segment. A random polarization rotation is applied to the signal between segments and within each segment the propagation of the signal is calculated through the following equations [9, 10]:

$$
\begin{aligned}
\frac{\partial A_{x}}{\partial z}+b \frac{\partial A_{x}}{\partial t} & +\frac{i}{2} \beta_{2} \frac{\partial^{2} A_{x}}{\partial t^{2}}+\frac{\alpha}{2} A_{x} \\
& =i \chi\left[\left|A_{x}\right|^{2}+\frac{2}{3}\left|A_{y}\right|^{2}\right] A_{x} \\
\frac{\partial A_{y}}{\partial z}-b \frac{\partial A_{y}}{\partial t} & +\frac{i}{2} \beta_{2} \frac{\partial^{2} A_{y}}{\partial t^{2}}+\frac{\alpha}{2} A_{y} \\
& =i \chi\left[\left|A_{y}\right|^{2}+\frac{2}{3}\left|A_{x}\right|^{2}\right] A_{y}
\end{aligned}
$$

where $A_{x}$ and $A_{y}$ are the complex envelopes of the x- and $\mathrm{y}$-polarized field components, $\beta_{2}$ is the group-velocity dispersion parameter, $\alpha$ is the attenuation constant, $\mathrm{z}$ is distance along the fiber segment, $t$ is the retarded time and $\chi$ is the nonlinear coefficient. PMD is included through the parameter $b$, with $b=\frac{d \beta_{x}}{d \omega}-\frac{d \beta_{y}}{d \omega}$, where $\beta_{x}$ and $\beta_{y}$ are the propagation constants for the $\mathrm{x}$ and $\mathrm{y}$ polarization components. $\beta_{2}$ is related to the dispersion parameter $\mathrm{D}$ by $\beta_{2}=\frac{-\lambda^{2}}{2 \pi c} D$.

For simulation of nonlinear propagation the (timeaveraged) optical power is set to $5 \mathrm{dBm}$ at the input of each transmission fiber and $-5 \mathrm{dBm}$ at the input of each DCF and the nonlinear coefficients of transmission fiber and DCF are $1.9 \mathrm{~W}^{-1} \mathrm{~km}^{-1}$ and $6.5 \mathrm{~W}^{-1} \mathrm{~km}^{-1}$, respectively. For the simulation of linear propagation, the nonlinear coefficients of the transmission fibers and DCFs are set to zero. The attenuation constants of transmission fiber and DCF are $0.046 \mathrm{~km}^{-1}$ and $0.115 \mathrm{~km}^{-1}$, respectively. Optical amplification in the simula- tions is noise free. The simulations evaluate the system penalties due to signal distortion, and the influence of optical and electrical noise is not included.

The receiver consists of a square-law photodetector and a fifth-order Bessel filter with 3-dB bandwidth of $7.5 \mathrm{GHz}$. System performance is quantified through the eye opening, defined as $I_{1}-I_{0}$, where $I_{1}$ is the minimum value of a 1-bit, and $\mathrm{I}_{0}$ is the maximum value of a 0 -bit, within a timing window with width equal to $15 \%$ of the bit period. The timing window is positioned within the eye such that the eye opening is maximized. Penalty for an eye opening $E_{1}$ relative to reference eye opening $E_{0}$ is defined as $10 \cdot \log \left(E_{0} / E_{1}\right)$.

In the absence of PMD, nonlinear propagation in system A results in a $0.2 \mathrm{~dB}$ improvement in eye opening relative to linear propagation, whereas system $B$ suffers a $1.4 \mathrm{~dB}$ penalty from nonlinearity. The PMD penalty is defined as the eye opening penalty with PMD relative to the same system configuration (e.g., nonlinear or linear propagation; system $\mathrm{A}$ or B) without PMD.

The PMD compensator structures are shown in Fig. (2). In Fig. (2a) is a single-stage compensator consisting of a polarization controller (PC) followed by a variable DGD element. In Fig. (2b) is a three-stage compensator made up of three variable DGD elements, each preceded by a PC. The PCs have two degrees of freedom (DOF) [11], so the singlestage compensator has three DOF and the three-stage compensator has nine.

a)

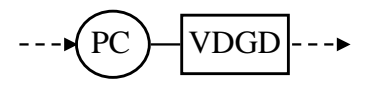

b)

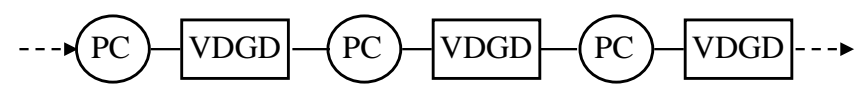

Fig. (2). PMD compensator structure with (a) single-stage compensation and (b) three-stage compensation. VDGD: variable DGD element.

Three different control methods are considered for the single-stage compensator: 1) the PC and the DGD of the compensator are set to maximize the DOP of the signal at the output of the compensator [12];2) the PC and DGD of the compensator are set to maximize the eye opening at the output of the compensator [13]; and 3) the compensator is controlled to compensate exactly the first-order PMD of the fiber (ideal first-order PMDC). A single control method is considered for the three-stage compensator: the polarization controllers and variable DGD elements are set to maximize the eye opening at the output of the compensator.

When the compensators are controlled to maximize the DOP or the eye opening, the particle swarm optimization technique [14] is used to search for the global optimum setting of the compensator.

\section{SIMULATION RESULTS WITHOUT PMDC}

The statistical variations inherent in PMD are accounted for by simulating ensembles of 2500 fibers. The mean DGD of the ensemble for system A is 35 ps, and for system B the 
mean DGD is 25 ps. System A with nonlinear propagation exhibits an increased tolerance to PMD. To clarify comparisons of PMDC with linear and nonlinear propagation in system A, a higher mean DGD value is used than in system B. For a given system (A or B), the same ensemble of fibers is used for all simulated configurations, allowing a direct comparison of the results with linear and nonlinear propagation as well as direct comparisons between different PMDC techniques.

The number of fibers used in the ensembles is not large enough to rigorously determine the impact of PMD at the low probability values that are normally of interest in system design. However, the ensemble size of 2500 fibers is large enough to allow observation of major differences in PMDC between linear and nonlinear propagation.

In linear NRZ systems the penalty from first-order PMD has a strong correlation to the parameter $\mathrm{K} \equiv \tau^{2} \gamma(1-\gamma)$, where $\tau$ is the fiber DGD and $\gamma$ reflects the relative power distribution in the fast and slow PSPs $(0 \leq \gamma \leq 1)$ [15]. Fig. (3) shows PMD penalties plotted $v s \mathrm{~K}$ in systems $\mathrm{A}$ and $\mathrm{B}$ with linear and nonlinear propagation. There are clearly large differences in PMD penalties between linear and nonlinear propagation, and, with nonlinear propagation, there are large differences in the behavior of the two systems, even allowing for the difference in mean DGD values.

The PMD penalties with linear propagation are strongly correlated with $\mathrm{K}$ indicating a strong correlation with first-

a)

Linear

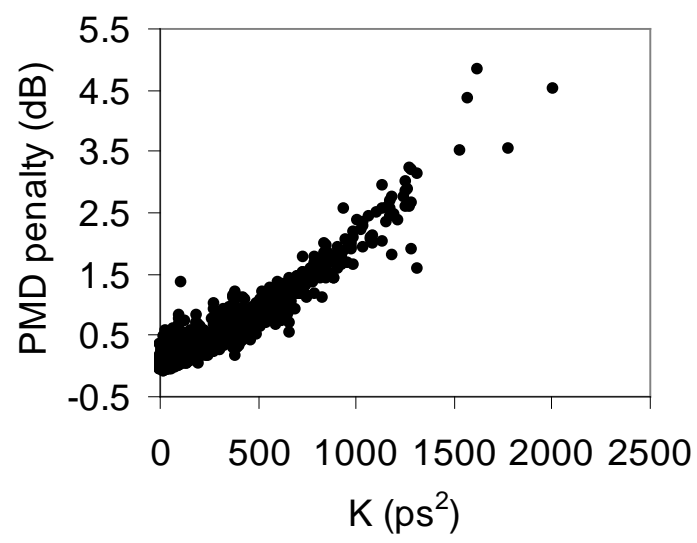

b)

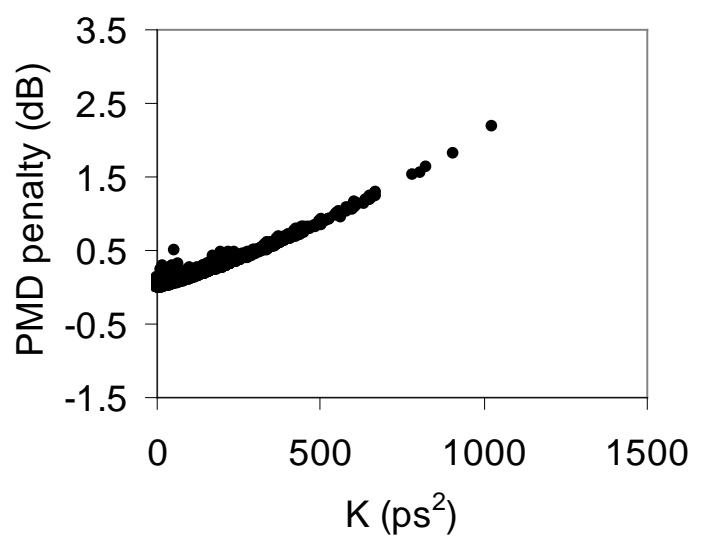

order PMD. For system B, the penalties with nonlinear propagation increase with $\mathrm{K}$ with a similar slope as in linear propagation. But there is much more scatter in the nonlinear case, with the possibility of both higher and lower penalties relative to the linear case. The maximum PMD penalty is higher with nonlinearity than without. The PMD penalties with nonlinear propagation in system A have a tendency to increase with $\mathrm{K}$, but the penalties are significantly lower than with linear propagation.

With nonlinear propagation in system B there are numerous instances of large negative penalty. This is not the case in system A. The main cause of the difference is the net dispersion in the two systems. The net dispersion of $300 \mathrm{ps} / \mathrm{nm}$ in system $\mathrm{A}$ is close to optimal (without PMD) for $5 \mathrm{dBm}$ launch power, while the net dispersion of $0 \mathrm{ps} / \mathrm{nm}$ in system $B$ results in a nonlinear penalty of $1.4 \mathrm{~dB}$. (The $1.4 \mathrm{~dB}$ nonlinear penalty in system B can be almost completely eliminated by increasing the net disperison from 0 to $300 \mathrm{ps} / \mathrm{nm}$ through adjustment of the post-compensation.) With favorable alignment of the input SOP, the interaction of higherorder PMD and nonlinear chirp can lead to a negative PMD penalty for system B $[3,6]$. This is illustrated in Fig. (4). The PMD penalty is plotted $v_{s} \boldsymbol{\Omega}_{\omega} \cdot \mathbf{S}_{\mathrm{in}}$, where $\boldsymbol{\Omega}$ is the input PMD vector in Stokes space, $\boldsymbol{\Omega}_{\omega}$ is the frequency derivative of $\boldsymbol{\Omega}$, and $\mathbf{S}_{\text {in }}$ is the Stokes vector of the signal polarization at the fiber input. The direction of $\boldsymbol{\Omega}$ is that of the slow input PSP of the fiber. Both $\boldsymbol{\Omega}$ and $\boldsymbol{\Omega}_{\omega}$ are defined at the carrier frequency of the signal.

\section{Nonlinear}
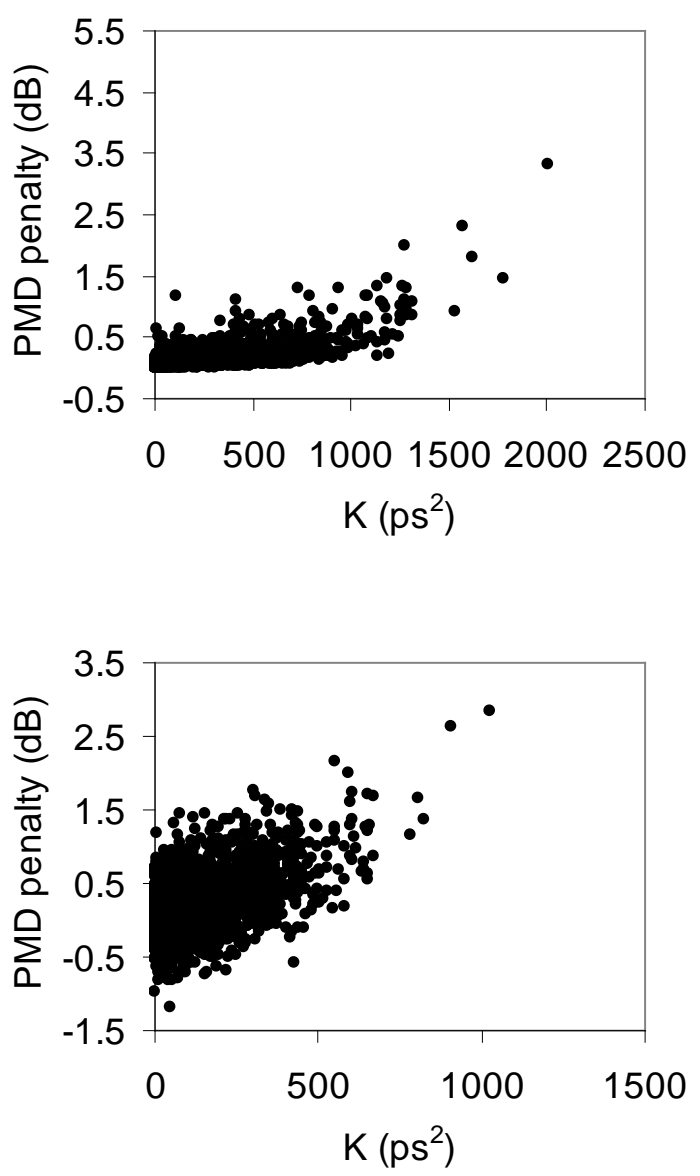

Fig. (3). PMD penalties $v s \mathrm{~K}$ with linear and nonlinear propagation for (a) system A and (b) system $\mathrm{B}$. 


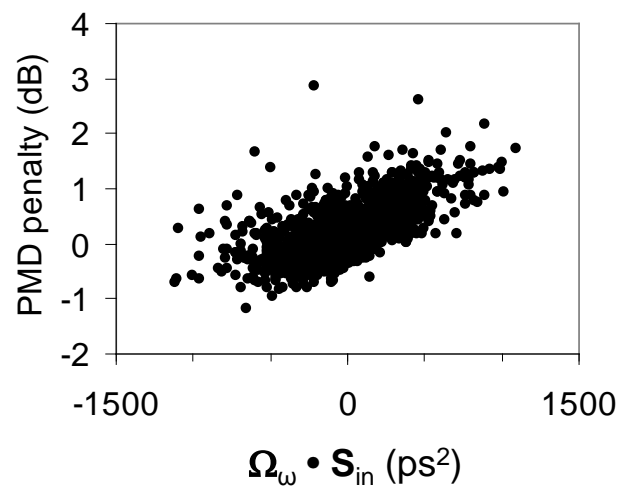

Fig. (4). PMD penalty $v_{s} \boldsymbol{\Omega}_{\omega} \cdot \mathbf{S}_{\text {in }}$ for system B with nonlinear propagation.

Fig. (5) shows DOP values plotted vs $\mathrm{K}$ in systems A and $\mathrm{B}$ with linear and nonlinear propagation. (The DOP is calculated for each fiber in an ensemble by averaging over the entire 64-bit pattern). As expected, the linear results show a strong negative correlation between the DOP and $\mathrm{K}$. The DOP is also negatively correlated with $\mathrm{K}$ for nonlinear propagation, but there is much more scatter in the DOP values and the DOP can be much lower than with linear propagation. With nonlinear propagation the signal undergoes a large spectral broadening which tends to result in lower DOP than in the linear case [12]. The extent of the spectral broadening is shown in Fig. (6).

a)

Linear

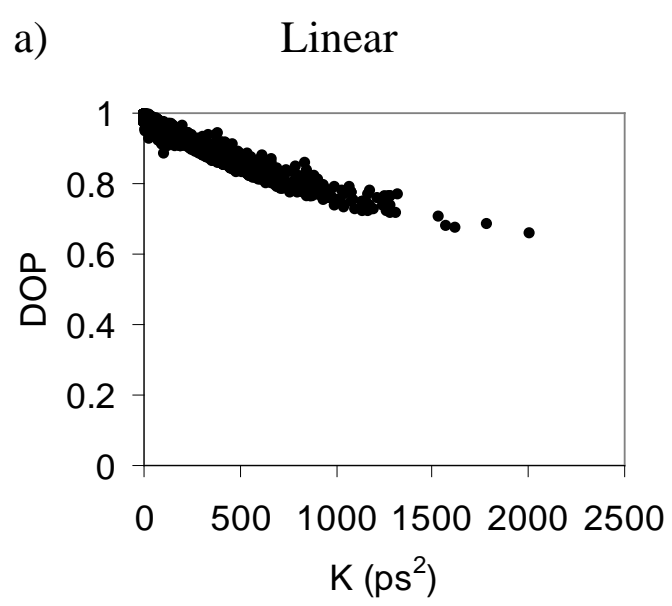

b)

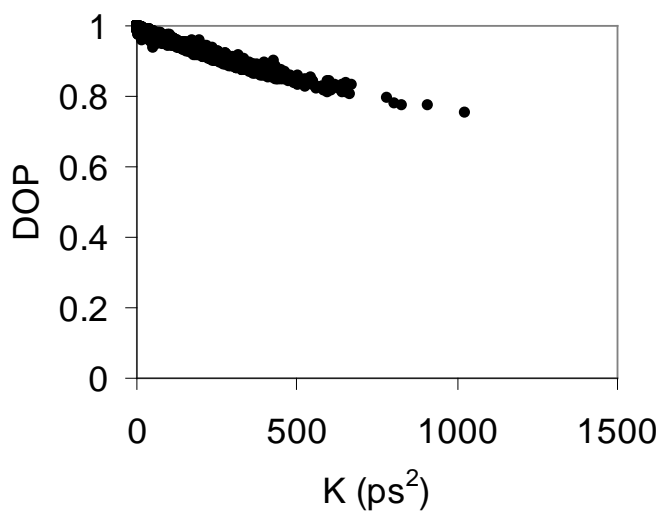

\section{SINGLE-STAGE PMDC}

Fig. (7) shows the distributions of the PMD penalties and DOP values for single-stage PMDC with optimization of the DOP and optimization of the eye opening. There are, for both control methods, clear differences between the results of PMDC with and without fiber nonlinearity. An analysis of PMDC for these systems based on linear propagation would not properly characterize PMDC in the nonlinear case.

Compensation based on optimization of the DOP leads to a reduction in PMD penalties with nonlinear propagation, but the residual penalties after compensation are higher than in the linear case, particularly for system B. The compensator is able to achieve significant increases in DOP with nonlinearity, although not to the same extent as with linear propagation.

Optimization of the eye opening will obviously be the best control method in our simulations, since the eye opening is used as the performance metric. The question is how large an improvement can be achieved by utilizing the eye opening. With nonlinear propagation, compensation through optimization of the eye opening does show significant improvement compared to compensation with optimization of the DOP. Similar to the results found in DMS systems in [8], we find that there are potentially large benefits obtainable by using a control method based on optimization of the eye opening with nonlinear propagation in NRZ systems.
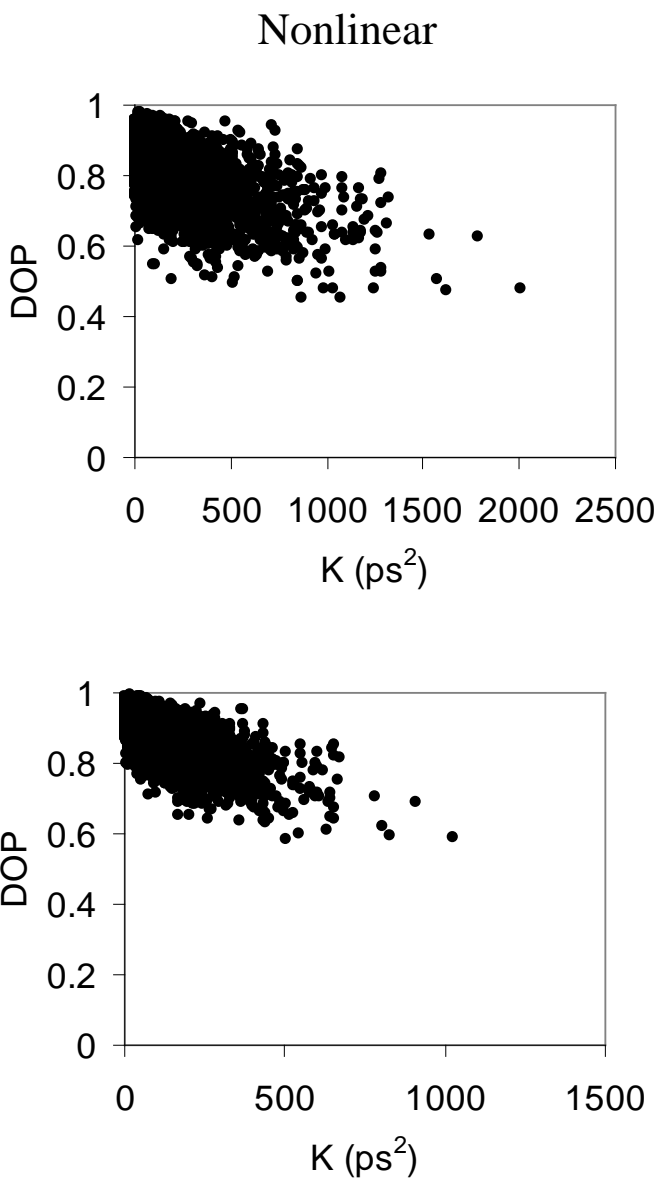

Fig. (5). DOP $v s \mathrm{~K}$ with linear and nonlinear propagation for (a) system A and (b) system B. 


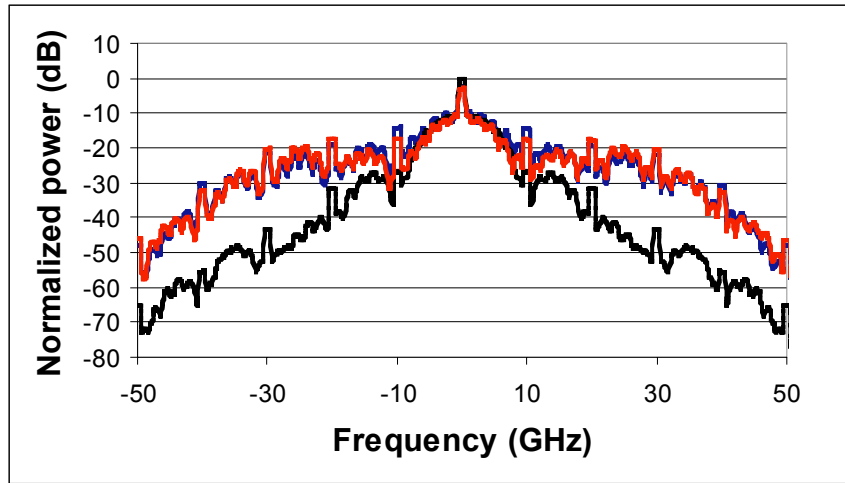

Fig. (6). Power spectrum of the optical signal at system output for linear propagation (black curve), nonlinear propagation in system A (red curve), and nonlinear propagation in system B (blue curve). PMD is set to zero in each case.

There is a possibility with nonlinear propagation that compensation based on optimization of the DOP could be improved by optical filtering of the monitor signal, as is the case in linear systems with large bandwidth (relative to NRZ) modulation formats [16]. We have not investigated that possibility in this work.

With linear propagation, compensation based on optimization of the eye opening tends to produce a large increase in the DOP, comparable to that given by optimization of the
DOP. In contrast, with nonlinear propagation, compensation based on optimization of the eye opening tends to produce lower DOP values than are found without compensation.

\section{IDEAL FIRST-ORDER AND ALL-ORDER PMDC}

Simulations in this section examine PMDC that is directly based on the PMD properties of the fiber. In ideal first-order PMDC, the DGD of the compensator is set equal to the fiber DGD and the PC is set so that a polarization state aligned with the fast output principal state of polarization (PSP) of the fiber is transformed to the slow axis of the compensator. (The fiber DGD and PSPs are defined at the carrier frequency of the signal).

In all-order PMDC the Jones matrix of the compensator, $\mathrm{U}^{-1}(\omega)$, is the inverse of the Jones matrix $\mathrm{U}(\omega)$ which describes linear propagation through the fiber. The compensator consists of the same number $(\mathrm{N})$ of segments as the fiber, and

$U^{-1}(\omega)=U_{1}^{-1}(\omega) U_{2}^{-1}(\omega) \cdots U_{N}^{-1}(\omega)$,

where $U_{n}(\omega)$ is the Jones matrix of the nth fiber segment and $U(\omega)=U_{N}(\omega) \cdots U_{2}(\omega) U_{1}(\omega)$.

Propagation in the all-order PMD compensator is linear, which is the case with all PMDC in this paper. With linear a)

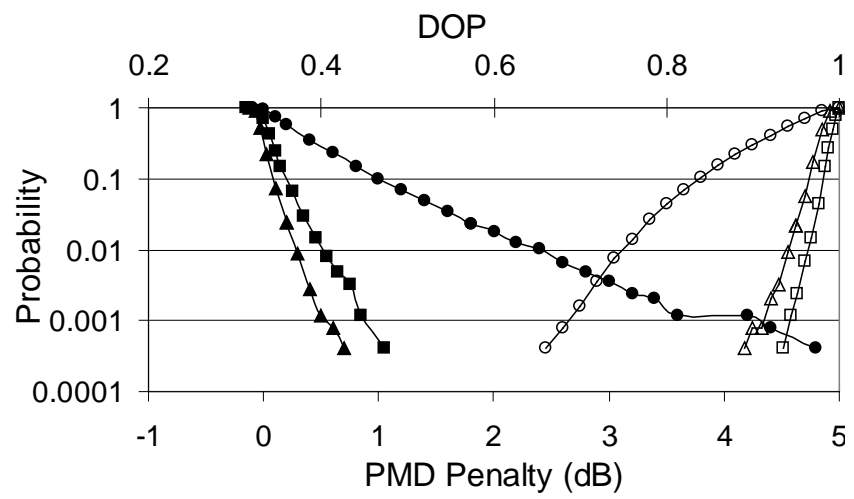

b)

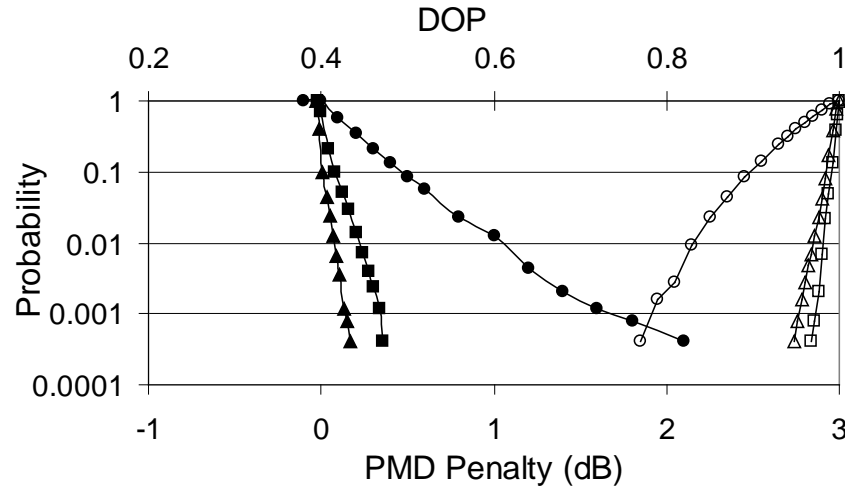

Nonlinear
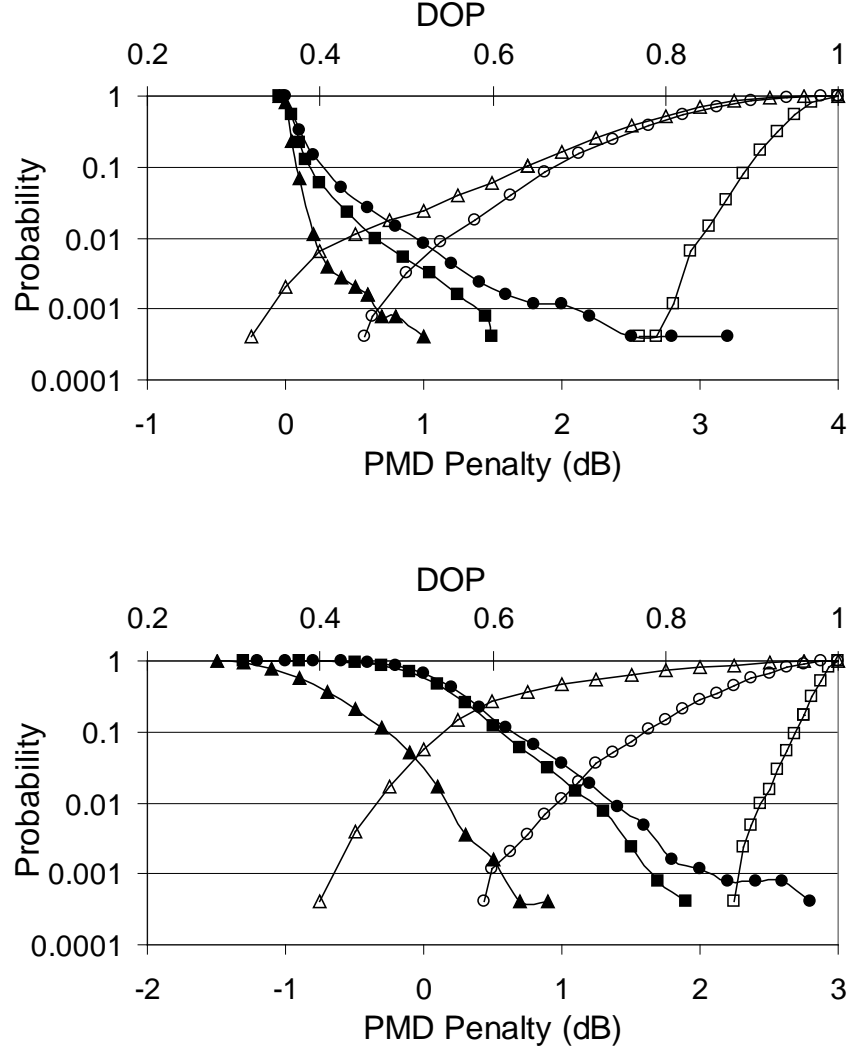

Fig. (7). Complementary cumulative probability of the PMD penalty (filled data points) and cumulative probability of the DOP (open data points) without PMD compensation (circles), with single-stage PMDC optimizing the DOP (squares), and with single-stage PMDC optimizing the eye opening (triangles). (a) System A with linear and nonlinear propagation; (b) System B with linear and nonlinear propagation. 
propagation in the transmission fiber and DCFs, all-order PMDC completely compensates the PMD such that there is no PMD penalty and the DOP is returned to 1 , the value at the input of the fiber.

Fig. (8) shows the distributions of the PMD penalties with ideal first-order PMDC and with all-order PMDC. Ideal first-order PMDC is clearly ineffective with the nonlinear propagation - there is much higher penalty after compensation than in the linear case. Whereas the DOP after compensation is returned close to 1 with linear propagation, in the nonlinear case the DOP is not, on the whole, improved by first-order PMDC. With nonlinear propagation and all-order PMDC, high penalties can still remain after the compensation and the signal DOP tends to be somewhat lower with the compensation than without.

The results with all-order PMDC reveal important information on PMD compensation in nonlinear systems. One potential strategy for improving PMDC with nonlinear propagation is to compensate higher orders of PMD, with the goal of mitigating the increase in signal spectral width caused by the nonlinearity. However, these results show that this is not necessarily a promising approach. In the systems studied here there were still significant residual penalties even after all orders of PMD were compensated. The spectral content of the signal continually changes with nonlinear propagation, and the penalty and the depolarization caused by PMD depend on the evolution of the PMD and the signal along the length of the system.

\section{THREE-STAGE PMDC}

We examine in this section a three-stage PMD compensator using optimization of the eye opening as control method. The simulation results are presented in Fig. (9). With linear propagation in systems A and B the PMD penalties are reduced to values below $0.05 \mathrm{~dB}$ and the DOP after compensation is increased to levels above 0.94. There is large improvement for system A compared to the results with single-stage compensation - the three-stage compensator is better able to cope with the higher-order PMD that is present with mean DGD of $35 \mathrm{ps}$.

Three-stage compensation is also very effective with nonlinear propagation. For system A, the maximum penalty after compensation is $0.12 \mathrm{~dB}$, comparable to the performance achieved with linear propagation. For system B, the maximum penalty after compensation is $-0.9 \mathrm{~dB}$. For all fibers in the ensemble the performance with PMDC is improved by at least $0.9 \mathrm{~dB}$ relative to the case where PMD is absent. The compensator operates such that the combined PMD of the fiber and compensator can partially compensate the $1.4 \mathrm{~dB}$ nonlinear penalty. To further demonstrate this point, we simulated a single instance (i.e., a single random seed for the optimization routine) of three-stage PMDC in
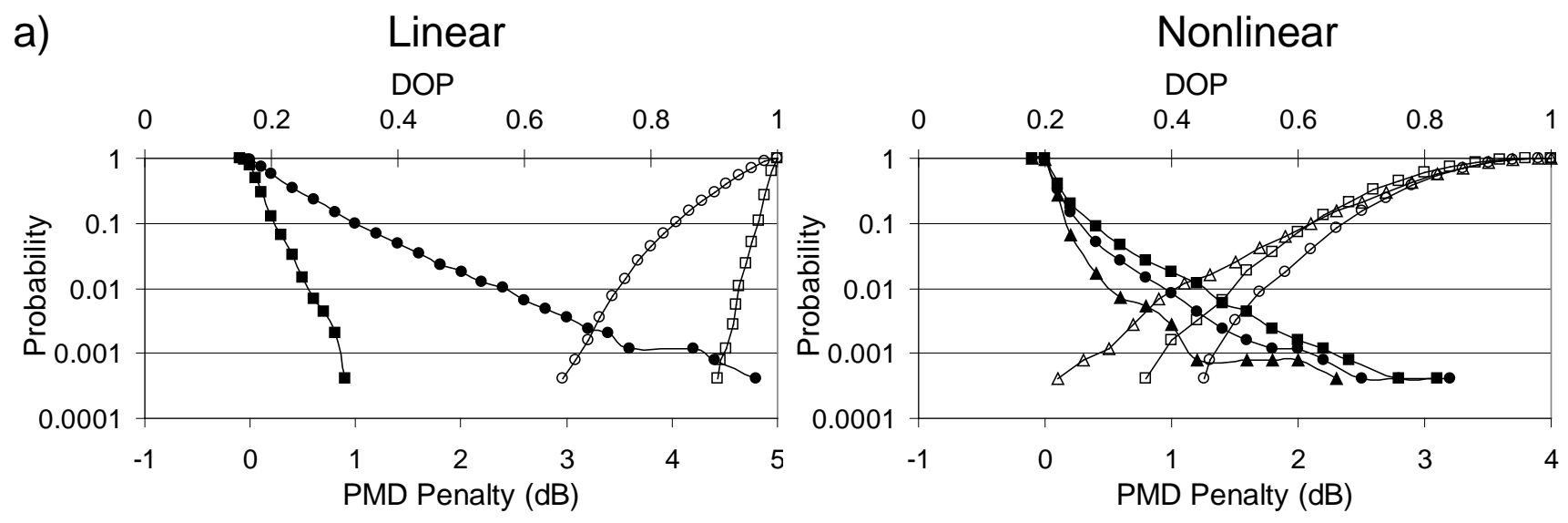

b)
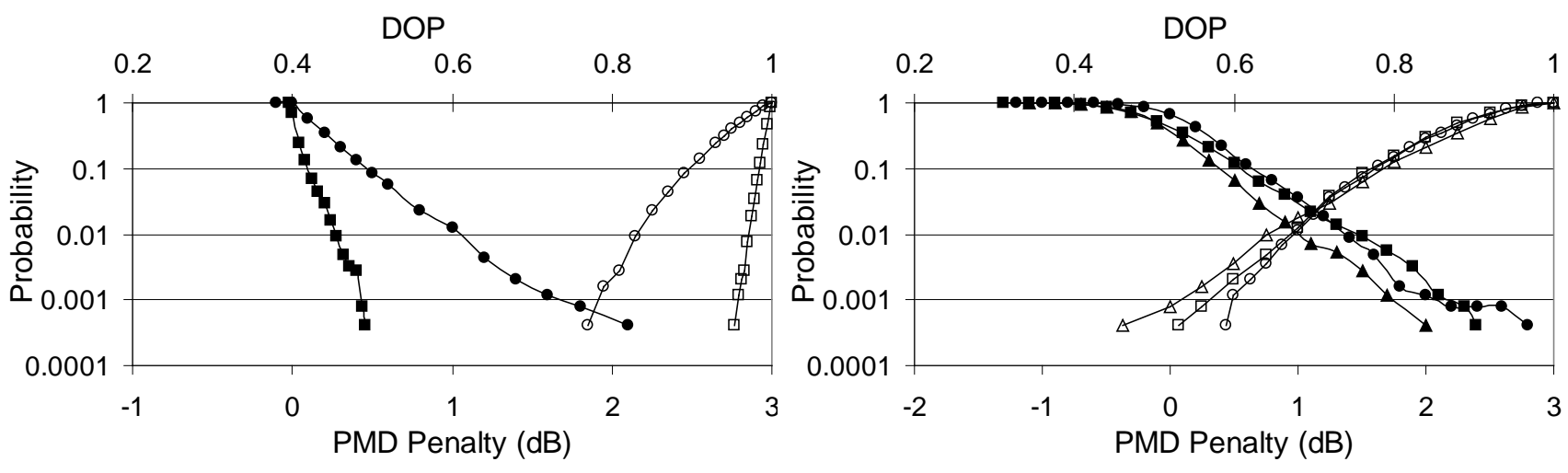

Fig. (8). Complementary cumulative probability of the PMD penalty (filled data points) and cumulative probability of the DOP (open data points) without PMD compensation (circles), with ideal first-order PMDC (squares), and with all-order PMDC (triangles). (a) System A with linear and nonlinear propagation; (b) System B with linear and nonlinear propagation. 

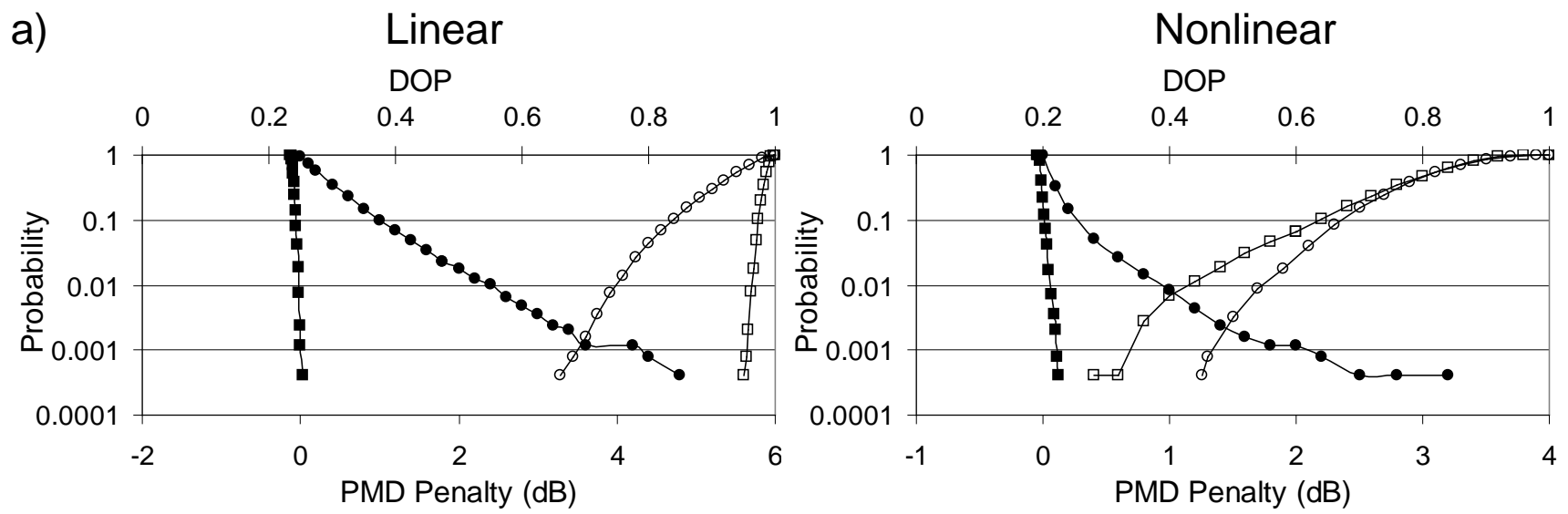

b)
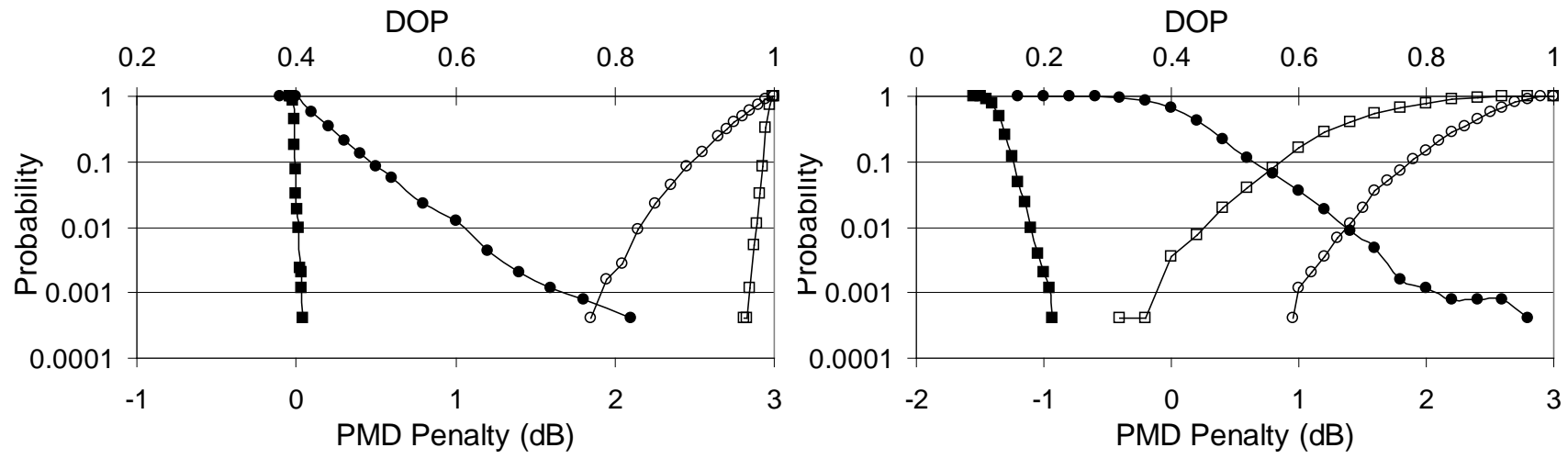

Fig. (9). Complementary cumulative probability of the PMD penalty (filled data points) and cumulative probability of the DOP (open data points) without PMD compensation (circles) and with three-stage PMDC optimizing the eye opening (squares). (a) System A with linear and nonlinear propagation; (b) System B with linear and nonlinear propagation.

system B with the fiber PMD set to zero. In this case the PMDC increased the eye opening by $1.3 \mathrm{~dB}$, almost completely compensating the nonlinear penalty. Three-stage compensation with optimization of the eye opening clearly offers large performance benefits with nonlinear propagation in the two systems studied, compared to the other PMDC techniques examined in this paper.

Similar to the results found with single-stage compensation, three-stage compensation with maximization of the eye opening tends to produce lower DOP values than are found without compensation.

An interesting outcome of the three-stage simulations is that with nonlinear propagation the compensator does not necessarily reduce first-order PMD. Table 2 compares the mean and maximum values of the parameter $\mathrm{K}$ with three stage PMDC to the values without PMDC. (For three-stage compensation $\mathrm{K}$ is specified for the combination of fiber plus compensator.) In system $\mathrm{B}$ the parameter $\mathrm{K}$ with nonlinear propagation actually tends to be larger with compensation than without. In system $\mathrm{A}$ the parameter $\mathrm{K}$ tends to be reduced by PMDC with nonlinear propagation but there can still be large values (e.g., compared to PMDC with linear propagation). This behavior of the three-stage compensator is consistent with results presented above, where compensation based on fiber PMD properties was ineffective with nonlinear propagation.
Table 2. Mean and Maximum Values of $\mathbf{K}$

\begin{tabular}{|c|c|c|c|c|}
\hline System & Compensation & Propagation & $\begin{array}{c}\text { Mean } \\
\mathbf{K}\left(\mathbf{p s}^{2}\right)\end{array}$ & $\begin{array}{c}\text { Maximum } \\
\mathbf{K}\left(\mathbf{p s}^{2}\right)\end{array}$ \\
\hline \hline A & Three-stage & Nonlinear & 232.2 & 1425.6 \\
\hline A & Three-stage & Linear & 36.2 & 307.2 \\
\hline A & None & $\begin{array}{c}\text { Linear or } \\
\text { nonlinear }\end{array}$ & 248.9 & 2007.0 \\
\hline B & Three-stage & Nonlinear & 260.2 & 1721.3 \\
\hline B & Three-stage & Linear & 5.7 & 173.5 \\
\hline B & None & $\begin{array}{c}\text { Linear or } \\
\text { nonlinear }\end{array}$ & 127.0 & 1024.0 \\
\hline
\end{tabular}

Further investigation is required to fully understand the behavior of the three-stage compensator in nonlinear systems and to determine whether similar performance could be obtained in a compensator with a reduced number of DOF, e.g., with a two-stage compensator.

\section{CONCLUSION}

The effect of single-channel nonlinearity on PMDC was investigated for a 2000-km NRZ system with $10 \mathrm{~Gb} / \mathrm{s}$ bit rate. Clear differences were found between the results of 
PMDC with and without nonlinearity for all the PMDC techniques that were examined. Analysis of PMDC in this system based on linear propagation would not properly characterize PMDC in the nonlinear case.

With nonlinear propagation, PMDC based directly on the first- and higher-order PMD of the fiber was ineffective. Ideal first-order compensation offered little benefit, and allorder PMDC showed only limited benefit.

Consistent with results found for a DMS system in [8], compensation based on optimization of the eye opening offered significant improvement with nonlinear propagation over compensation based on optimization of the DOP. For first-order PMDC, all-order PMDC, and PMDC based on optimization of the eye opening, the compensation did not generally lead to increased values of DOP. This is much different behavior than is found with linear propagation.

Three-stage compensation with optimization of the eye opening showed a large improvement in performance with nonlinear propagation compared to the other PMDC techniques examined in this paper. With the dispersion of the system detuned from the optimum operating point, threestage compensation resulted in negative PMD penalties for all fibers in the simulated ensemble. Further investigation is required to determine whether similar performance could be obtained in a compensator with a reduced number of DOF, e.g., with a two-stage compensator.

\section{ACKNOWLEDGEMENTS}

The authors acknowledge financial support from the $\mathrm{Ca}$ nadian funding agencies: Discovery Grant of NSERC (Natural science and engineering research council of Canada), and the Centres of excellence program of NSERC: AAPN (Agile All Photonics Networks).

\section{REFERENCES}

[1] Takahashi T, Imai T, Aiki M. Automatic compensation technique for timewise fluctuating polarization mode dispersion in in-line amplifier systems. Electron Lett 1994; 30: 348-9.
[2] Winters JH, Hass Z, Santoro MA, Gnauck AH. Optical equalization of polarization dispersion. In: Proc Soc Photo-Opt Instr Eng; 1992; pp. 346-7.

[3] Ibragimov E, Menyuk CR, Kath W. PMD-induced reduction of nonlinear penalties in terrestrial optical fiber transmission. In: Proc Conf Opt Fiber Commun; Baltimore, MD: 2000; Paper WL3.

[4] Möller L, Boivin L, Chandrasekhar S, Buhl L. Setup for demonstration of cross-channel induced nonlinear PMD in WDM system. Electron Lett 2001; 37: 306-8.

[5] Khosravani R, Xie Y, Yan LS, Willner AE, Menyuk CR. Limitations to first-order PMD compensation in WDM systems due to XPM-induced PSP changes. In: Proc Conf Opt Fiber Commun; Anaheim, CA: 2001; Paper WAA5.

[6] Menyuk CR, Marks BS. Interaction of polarization mode dispersion and nonlinearity in optical fiber transmission systems. J Lightwave Technol 2006; 24: 2806-26.

[7] Karlsson M, Sunnerud H. Effects of nonlinearities on PMDinduced system impairments. J Lightwave Technol 2006; 24: 412737.

[8] Alzetta D, Matsumoto M. Transmission degradation due to polarization-mode dispersion in linear and nonlinear systems. In: Proc Conf Opt Fiber Commun; Anaheim, CA: 2002; Paper TuI3.

[9] Marcuse D, Menyuk CR, Wai PKA. Application of the ManakovPMD equation to studies of signal propagation in optical fibers with randomly varying birefringence. J Lightwave Technol 1997; 15: $1735-46$

[10] Khosravani R, Willner AE. System performance evaluation in terrestrial systems with high polarization mode dispersion and the effect of chirping. IEEE Photon Technol Lett 2001; 13: 296-8.

[11] Heismann F. Analysis of a reset-free polarization controller for fast automatic polarization stabilization in fiber-optic transmission systems. J Lightwave Technol 1994; 12: 690-9.

[12] Kikuchi N. Analysis of signal degree of polarization used as control signal for optical polarization mode dispersion compensation. J Lightwave Technol 2001; 19: 480-6.

[13] Buchali F, Lanne S, Thiéry JP, Baumert W, Bülow H. Fast eye monitor for $10 \mathrm{Gbit} / \mathrm{s}$ and its application for optical PMD compensation. In: Proc Conf Opt Fiber Commun; 2001: Anaheim, CA; Paper TuP5.

[14] Zhang X, Zheng Y, Shen Y, Zhang J, Yang B. Particle swarm optimization used as a control algorithm for adaptive PMD compensation. IEEE Photon Technol Lett 2005; 17: 85-7.

[15] Kogelnik H, Jopson RM, Nelson LE. Polarization-Mode Dispersion. In: Kaminow IP, Li T, Eds. Optical Fiber Telecommunications IV-B: Systems and Impairments, Academic Press, San Diego, 2002; pp. 725-861.

[16] Xie C, Möller L. Comparison of different feedback signals for onestage polarization-mode dispersion compensators. IEEE Photon Technol Lett 2005; 17: 570-2. 